\title{
Centro de Atenção Psicossocial Álcool e Outras Drogas Regional: Potencialidades e
}

\section{Obstáculos}

\author{
Psychosocial Attention Center Alcohol and Other Regional Drugs: Potentiality and Obstacles \\ Alcohol y Otros Centros de Atención Psicosocial Regional: Potencialidades y Obstáculos
}

Recebido: 03/01/2022 | Revisado: 09/01/2022 | Aceito: 15/01/2022 | Publicado: 17/01/2022

\author{
Karine Lucero Carvalho \\ ORCID: https://orcid.org/0000-0001-9558-7155 \\ Hospital Casa de Saúde, Brasil \\ E-mail: karine.lucero2016@gmail.com \\ Liane Beatriz Righi \\ ORCID: https://orcid.org/0000-0002-1614-8793 \\ Universidade Federal de Santa Maria, Brasil \\ E-mail: lianerighi@gmail.com \\ Maria Carolina da Costa Pinheiro \\ ORCID: https://orcid.org/0000-0003-1567-2347 \\ Universidade Federal de Pelotas, Brasil \\ E-mail: maria-meireles@saude.rs.gov.br \\ Ana Luiza Ferrer \\ ORCID: https://orcid.org/0000-0003-0862-1015 \\ Universidade Federal de Santa Maria, Brasil \\ E-mail: aluizaferrer@gmail.com \\ Cristiane Trivisiol Arnemann \\ ORCID: https://orcid.org/0000-0003-2684-3068 \\ Universidade Federal de Santa Maria, Brasil \\ E-mail: cris.trivisiol@gmail.com \\ Diogo Faria Corrêa da Costa \\ ORCID: https://orcid.org/0000-0001-7720-5993 \\ Secretaria Estadual da Saúde da Rio Grande do Sul, Brasil \\ E-mail: diogo-costa@saude.rs.gov.br \\ Daiana Foggiato de Siqueira \\ ORCID: https://orcid.org/0000-0002-8592-379X \\ Universidade Federal de Santa Maria, Brasil \\ E-mail: daianasiqueira@yahoo.com.br
}

\begin{abstract}
Resumo
Os dispositivos que ordenam o cuidado em território preconizado pela Lei da Reforma Psiquiátrica são os Centros de Atenção Psicossocial. Em quatro décadas do início do movimento antimanicomial muitos avanços ocorreram, porém hoje se percebe a descredibilidade com a Política de Saúde Mental nas esferas de governo, retrocedendo nas formas de tratamento. Tal cenário potencializa este estudo que tem por objetivo avaliar um Centro de Atenção Psicossocial álcool e outras drogas regional a partir das potencialidades e os obstáculos identificados pelos diferentes grupos de interesse. De cunho qualitativo se utilizou a Avaliação de Quarta Geração. O período da coleta foi entre maio e novembro de 2018 por meio de pesquisa documental, entrevistas semiestruturadas e grupos focais. Realizou-se seleção por grupos de interesse para definir os participantes: gestores, trabalhadores e usuários. Os núcleos temáticos foram: a configuração regional, a intersetorialidade e o matriciamento. A conclusão foi que a regionalização, utilizada como estratégia de gestão, necessita de repactuações regulares entre os gestores para assegurar o funcionamento e a assistência ao tratamento; a intersetorialidade e o matriciamento, facilitadores de um cuidado ampliado, tem na distância e na definição de papéis dos serviços da rede seus maiores obstáculos.
\end{abstract}

Palavras-chave: Saúde mental; Serviços de saúde mental; Regionalização.

\begin{abstract}
The device that orders care in the territory recommended by the Psychiatric Reform Law is the Psychosocial Care Centers. In the four decades since the beginning of the anti-asylum movement, many advances have taken place, but today it is possible to perceive the lack of credibility with the Mental Health Policy in the spheres of government, going back in the forms of treatment. This scenario enhances this study, which aims to evaluate a regional Psychosocial Care Center for alcohol and other drugs based on the potential and obstacles identified by different interest groups. Of a qualitative nature, the Fourth Generation Assessment was used. The collection period was between May and November 2018 through documentary research, semi-structured interviews and focus groups. Interest groups were selected to define the participants: managers, workers and users. The thematic groups were: the
\end{abstract}


regional configuration, intersectoriality and matrix support. The conclusion was that regionalization, used as a management strategy, needs regular renegotiations between managers to ensure the functioning and assistance to the treatment; intersectoriality and matrix support, facilitators of expanded care, have the greatest obstacles in the distance and in the definition of roles of network services.

Keywords: Mental health; Mental health Services; Regionalization.

\section{Resumen}

El dispositivo que ordena la atención en el territorio recomendado por la Ley de Reforma Psiquiátrica son los Centros de Atención Psicosocial. En cuatro décadas desde el inicio del movimiento anti-asilo, se han producido muchos avances, pero hoy podemos ver la incredulidad de la Política de Salud Mental en los ámbitos gubernamentales, retrocediendo en términos de tratamiento. Este escenario potencia este estudio, que tiene como objetivo evaluar un Centro de Atención Psicosocial regional para el alcohol y otras drogas, en base a los potenciales y obstáculos identificados por diferentes grupos de interés. De carácter cualitativo, se utilizó la Evaluación de Cuarta Generación. El período de recolección fue entre mayo y noviembre de 2018 a través de investigación documental, entrevistas semiestructuradas y grupos focales. La selección se llevó a cabo por grupos de interés para definir los participantes: gerentes, trabajadores y usuarios. Los núcleos temáticos fueron: configuración regional, intersectorialidad y soporte matricial. La conclusión fue que la regionalización, utilizada como estrategia de gestión, requiere renegociaciones periódicas entre los administradores para asegurar el funcionamiento y la asistencia del tratamiento; La intersectorialidad y el soporte matricial, que facilitan la atención ampliada, tienen los mayores obstáculos en la distancia y en la definición de los roles de los servicios de red.

Palabras clave: Salud mental; Servicios de salud mental; Regionalización.

\section{Introdução}

O ano de 2019 é uma data importante para a Política de Saúde Mental brasileira, pois além de demarcar 41 anos do início do movimento antimanicomial no país e os 18 anos da Lei 10.216/2001 conhecida como a Lei da Reforma Psiquiátrica é o ano em que é deferido pelo governo federal uma nota técnica que legitima a volta dos hospitais psiquiátricos, dos ambulatórios de saúde mental e insere as comunidades terapêuticas na rede de atenção psicossocial (Brasil, 2001a; Brasil, 2019).

Desde o início do movimento antimanicomial liderado por psiquiatras e demais profissionais que trabalhavam nos hospitais psiquiátricos no final da década de 70 no Brasil, se buscava o fechamento gradual destes locais de tratamentos "desumanos" e redirecionar o tratamento de pessoas com transtornos mentais para o território. Nas décadas seguintes o movimento ganha força e impulsiona à Reforma Psiquiátrica (RP) Brasileira (Amarante, 2010).

Nos últimos anos muitos avanços ocorreram na Política de Saúde Mental brasileira como a diminuição de leitos psiquiátricos (80 mil na década de 1970 para 25.988 em 2014), o investimento em serviços de atenção psicossocial (mais de 2000 Centros de Atenção Psicossocial em 2014 com cobertura de 0,86 serviços por 100 mil/habitantes) e a contenção dos gastos com hospitais (de 75,24\% em 2002 para 20,61\% em 2013). Em contrapartida, os gastos com a atenção psicossocial passam de 24,76\% para 79,39\% até 2014, há registro de 610 Serviços Residenciais Terapêuticos (SRT's) com 2.031 moradores beneficiados, o Programa de Volta Para Casa passou a ter 4.349 beneficiários e as iniciativas de geração de renda chegaram a 1.008 ações (Brasil, 2002; 2011a; 2003; Amarante \& Nunes, 2018).

E apesar do progresso há um desafio atual que se impõe à reforma psiquiátrica, seus princípios e ideais não vêm sendo acolhidas como prioridade nos governos das três esferas (federal, estadual e municipal) nos últimos anos. O que culmina na falta de incentivo dificultando a execução de serviços promotores de vida (Souza \& Jorge, 2019). Para Amarante e Nunes (2018) não havia como negar o perigo de retrocessos em todo o processo construído ao longo de décadas no âmbito da RP, diante destes governos, o que acarretou com o resgate do modelo manicomial.

Diante deste cenário este estudo se torna pertinente ao reafirmar para a sociedade a relevância dos serviços especializados, Centros de Atenção Psicossocial (CAPS), como forma de cuidado na rede. Principalmente por apresentar um serviço que tem a premissa de regionalização de um serviço territorial na perspectiva de um cuidado não manicomial, logo no momento em que é deferido o retorno do cuidado ambulatorial por equipes multiprofissionais de atenção especializada em 
saúde mental, deslegitimando o apoio matricial e o trabalho intersetorial dos CAPS com os serviços de saúde e sócio assistenciais (Brasil, 2019).

Sendo que atualmente estão habilitados e em funcionamento no Brasil, aproximadamente 2.209 CAPS, destes 189 CAPS se encontram no Rio Grande do Sul, e deste conjunto, 29 na configuração regional (Brasil, 2015; Rio Grande Do Sul, 2016a). Frente ao descaso com o cuidado realizado em rede e ao retrocesso posto, há que se fortalecer tais serviços e trazer à reflexão: quais são as potencialidades e os obstáculos apresentados pelo CAPS regional para o fortalecimento da Rede de Atenção Psicossocial? E a partir deste interesse, buscou-se avaliar um Centro de Atenção Psicossocial Álcool e Outras Drogas Regional a partir das potencialidades e dos obstáculos identificados pelos diferentes grupos de interesse.

\section{Metodologia}

O presente estudo trata-se de uma pesquisa qualitativa com abordagem da avaliação de quarta geração. Por sua característica responsiva e construtivista a Avaliação de Quarta Geração é a metodologia pertinente a este estudo por permitir que os envolvidos compartilhem reflexões, opiniões, trabalhem os conflitos, partilhem decisões e que contribuam para o empoderamento destes (Guba \& Lincoln, 2011; Righi \& Gonçalves, 2018). Metodologia que favorece um delineamento teórico-metodológico sistemático, estruturado em passos que se desenham com base na lógica dialética e da interpretação hermenêutica (Kantorski et al., 2009; Kantorski et al., 2011).

A coleta ocorreu de maio a novembro de 2018 a partir da realização de pesquisa documental, grupos focais (GF) e entrevistas semiestruturada. Os cenários do estudo foram a $4^{\text {a }}$ Coordenadoria Regional de Saúde (4 ${ }^{\mathrm{a}} \mathrm{CRS}$ ) e o Centro de Atenção Psicossocial Álcool e Outras Drogas Regional Padre Otávio Ferrari (CAPS AD Pe. Otávio Ferrari), sendo que no primeiro espaço se deu uma das entrevistas semiestruturadas e uma parte da pesquisa documental, as demais entrevistas, os GF e a continuidade da pesquisa documental foram no segundo campo.

Dos 19 documentos avaliados (relatórios de atividades, ofícios, memorandos, relatório de visitas institucionais, formulário do Sistema Único de Saúde (FORMSUS) e atas) dois foram os documentos principais que embasaram o estudo, pela sua relevância no cenário: Projetos Institucionais (PI) do CAPS AD Pe. Otávio Ferrari de 2003 (implantação) e uma atualização de 2016 (Nova Palma, 2003; Bertoldo, 2016). A seleção dos participantes foi sustentada pelo conceito de grupos de interesse. Grupos de interesse são aqueles que, por definição, têm algum interesse no sujeito-objeto da avaliação (Righi; Gonçalves, 2018).

Nesse caso da avaliação do CAPS AD Pe. Otávio Ferrari, os grupos de interesses identificados foram: gestores, trabalhadores e usuários. O grupo de interesses gestores foi representado pela Secretária municipal de saúde, a Coordenadora da Política de Saúde Mental da $4{ }^{a}$ CRS (época da implantação) e a Coordenadora do serviço, e realizou-se a coleta por meio de entrevistas semiestruturada com a utilização de um roteiro com cinco questões norteadoras. Já os Grupos de interesses dos trabalhadores e usuários foram a partir de Grupo Focais organizados em GF dos trabalhadores e GF dos usuários, com um encontro de cada grupo com aproximadamente uma hora de duração.

Entende-se por grupo focal, técnica que possibilita a análise de dados obtidos numa situação de interação grupal, verificando de que modo às pessoas avaliam uma experiência, como definem um problema e como suas opiniões, sentimentos e representações encontram-se associados a determinado fenômeno do seu próprio ponto de vista (Figueiredo \& Campos, 2009; Backes et al., 2011). Neste caso como avaliam o CAPS AD Regional Pe. Otávio Ferrari.

Ao todo participaram 19 pessoas (03 da gestão, 05 trabalhadores e 11 usuários) identificadas pelas letras que correspondem ao seu grupo de interesse: Gestores (G); Trabalhadores (T) e Usuários (U) seguidos de um número arábico (exemplo: Trabalhador 1 - T1, Trabalhador - T2...). Foram utilizados recursos de áudio para gravação das falas dos participantes após autorização dos mesmos. Salienta-se que os vícios de linguagem foram corrigidos durante a transcrição. 
Para o delineamento dos núcleos temáticos foi utilizado o método do círculo hermenêutico dialético. Hermenêutico porque é interpretativo e dialético porque representa a comparação e o contraste das visões divergentes com a visão para a realização de um alto nível de síntese (Guba \& Lincoln, 2011). Tarjetas foram utilizadas como disparadoras do círculo, ou seja, das transcrições das entrevistas semiestruturadas do primeiro grupo de interesse formado pelos gestores se elaborou tarjetas com palavras chaves para serem disparadores de discussão no grupo subsequente e assim por diante com os grupos focais dos trabalhadores e usuários.

Por fim, a avaliação aconteceu a partir da discussão dos núcleos temáticos que correspondem ao objetivo do estudo referente às potencialidades e os obstáculos identificados pelos diferentes grupos de interesse. Os princípios éticos da pesquisa com seres humanos foram respeitados conforme a Resolução n ${ }^{\circ}$ 466/2012 do Conselho Nacional de Saúde, de modo a proteger os direitos dos participantes (Brasil, 2012). A pesquisa foi aprovada pelo Comitê de ética em pesquisa da instituição sob o número 2.711.187 e CAAE: 90751518.1.0000.534.

\section{Resultados e Discussão}

Foram avaliados três núcleos temáticos de forma a nortear a discussão: configuração regional; Intersetorialidade e matriciamento.

\section{Configuração regional}

Referente a configuração regional, primeiramente faz-se necessário caracterizar a região e o município sede do serviço. Nova Palma, cidade onde está localizado o CAPS AD Pe. Otávio Ferrari, foi fundada em 29 de julho de 1960 na região central do estado, tem uma população aproximada de 6.342 habitantes (IBGE, 2010).

A cidade faz parte da microrregião da Quarta colônia, juntamente com os municípios: Faxinal do Soturno, Agudo, Dona Francisca, Pinhal Grande, Restinga Seca, São João do Polêsine, Silveira Martins, Ivorá e Paraíso do Sul (Saad et al., 2012). A agropecuária, o comércio e a indústria são as principais atividades econômicas do município (Nova Palma, 2019).

Em janeiro de 2003 com o intuito de instituir um serviço de internação para tratamento de pessoas com transtornos mentais e comportamentais derivado do uso de álcool e outras drogas, é implantado na região central do estado, o Serviço Regional de Atenção à Saúde Mental - álcool e outras drogas (SERAISM-AD) no Hospital Nossa Senhora da Piedade de Nova Palma, com 13 leitos para internação (Nova Palma, 2003; Bertoldo, 2016).

No mesmo ano, para qualificar o tratamento iniciado na internação, é instaurado o CAPS AD Pe. Otávio Ferrari. Conforme o PI 2016: “o CAPS AD foi criado em 2003 em conjunto com o SERAISM-AD, onde havia uma equipe multiprofissional atendendo os mesmos usuários, ou seja, nos turnos diurnos permaneciam nos CAPS AD, enquanto que à noite ficavam sob o olhar da equipe de enfermagem do SERAISM-AD" (Bertoldo, 2016, p. 01). Durante o dia os usuários internados no SERAISM-AD davam continuidade ao tratamento no CAPS AD Pe. Otávio Ferrari.

Assim compreende-se que o CAPS AD Pe. Otávio Ferrari é implantado concomitante a criação dos leitos no hospital geral de Nova Palma, conforme:

Essa foi uma proposta bem interessante, ela começou com uma proposta de um serviço multiprofissional dentro de um Hospital, só que faltou financiamento do Estado. Então a proposta que a gente encontrou junto ao Ministério foi fazer isso, o hospital passou a ter os leitos de retaguarda para o CAPS, tanto que ele foi o primeiro CAPS, e já nasceu regional e $A D$ porque nós precisávamos atender essa população. Tinha uma demanda, era assustadora, a gente não tinha aonde, começou mais com alcoolismo depois entrou as drogas, e a gente não tinha leito e a gente precisava um suporte de internação. (G1) 
O PI 2003 apresenta uma forma de organização que se dava o cuidado: "os usuários internados participarão das atividades no SERAISM-AD, ora no CAPS AD, já que este último propiciará um processo de transição para ala hospitalar, vinculando os usuários do CAPS AD para que ocorra a manutenção do tratamento" (Nova Palma, 2003, p.03). Confirmado na fala do GF dos trabalhadores:

O CAPS AD foi estruturado para dar suporte à internação, então durante o dia passavam no CAPS AD, pra almoçar, tomavam medicação e dormiam no hospital, a equipe do CAPS era responsável pela alta. (T1)

Nota-se que a implantação do SERAISM-AD e de um CAPS AD foi impulsionado pela demanda crescente do uso dessas substâncias na região, naquele período. Tal evento é citado no PI 2003 como um dos três fatores que motivaram à implantação dos dois serviços: o fenômeno do êxodo rural ocasionado pela crise do modelo exportador e a mecanização da agricultura na região, o uso indiscriminado de benzodiazepínicos pelos índices elevados de transtornos depressivos e de ansiedade da população, além da cultura do consumo de álcool, principalmente o vinho, por tratar-se de uma região descendentes de italianos (Nova Palma, 2003).

Assim, vinculado ao SERAISM-AD, o CAPS AD inicia suas atividades à mesma área de abrangência do hospital, em vista que se tratava dos mesmos usuários provindos dos 29 municípios que faziam parte da $4^{\mathrm{a}}$ CRS. A regionalização está assegurada na forma de organização do Sistema Único de Saúde (SUS), disposto no Decreto No 7508/2011 que ainda dispõe sobre a forma de planejamento da saúde, da assistência à saúde, da articulação interfederativa, além de outras providências (Brasil, 2011b).

O decreto define dois parâmetros importantes para justificar a forma de organização regional: Região de Saúde e Redes de Atenção à Saúde (RAS). A Região de Saúde definida como espaço geográfico contínuo constituído por agrupamentos de municípios limítrofes, delimitado a partir de identidades culturais, econômicas e sociais, de redes de comunicação e infraestrutura de transportes compartilhados com a finalidade de integrar a organização, o planejamento e a execução de ações e serviços de saúde. A RAS considerada o conjunto de ações e serviços de saúde articulados em níveis de complexidade crescente, com a finalidade de garantir a integralidade da assistência à saúde (Brasil, 2011c).

Observa-se que o decreto no estado foi deferido em 2012, porém em 2003 a região da quarta colônia já se articulava em uma configuração que correspondesse às necessidades do cuidado em saúde mental deste território, mobilizando os recursos disponíveis para um mesmo fim. Isto se deve aos avanços da descentralização do SUS na década de 90 (séc. XX), onde por meio das Normas Operacionais Básicas (NOB) criaram-se as condições para que os municípios se constituíssem como os principais executores das ações e serviços de saúde (Teixeira, 2002).

Tal prática, no entanto, evidenciou que nenhum nível de forma isolada apresenta condições de realizar assistência em saúde em todas as suas complexidades, sendo o de maior complexidade inatingível, por não estar presente em todos os municípios. E em 2001 a NOB 96 é substituída pela Norma Operacional da Assistência à Saúde (NOAS) 2001 que vem enfatizar a cooperação e recupera a noção de economia de escala como fundamento para a racionalização (e a reconcentração) de recursos, resgatando a possibilidade de se construir sistemas de serviços de saúde que ultrapassem os limites territoriais dos municípios, em sua maioria de pequeno porte (Brasil 2001b; Teixeira, 2002).

Organizada por microrregiões, pode corresponder a apenas um município ou incluir vários outros de pequeno porte, cuja capacidade instalada tomada em conjunto corresponda ao nível de resolutividade, contemplando desde a atenção primária a alta complexidade (Brasil, 2001b). Desta forma, a regionalização tornou-se o meio para a construção de um sistema que contempla os serviços, conhecimentos e tecnologias para qualificar e garantir o acesso à atenção à saúde, buscando uma perspectiva concreta de ampliação e organização da rede, indo ao encontro da necessidade da população da região (Teixeira, 
2002).

Nesse caso, a atenção psicossocial da região se organiza de forma regional para contemplar os serviços da atenção primária à alta complexidade, ou seja, da atenção básica, passando pelo serviço especializado (CAPS AD) e as internações na alta complexidade. A habilitação pelo Ministério da Saúde do CAPS AD Pe. Otávio Ferrari foi em 05 de julho de 2005, sendo oficialmente em 2011 quando as equipes se desmembram e cada serviço passa a se organizar com equipes multiprofissionais e funções próprias.

Em 2011 passa a ser constituído de forma microrregional, em vista que sua área de abrangência se delimita aos nove municípios que fazem parte da Quarta Colônia, não mais a toda área da $4^{\mathrm{a}}$ CRS (Bertoldo, 2016, p. 02). Cabe ressaltar, porém que o nome fantasia "Regional" permanece até hoje pela sua história e como culturalmente é conhecido na região.

Esta nova configuração microrregional, tem a premissa de corresponder ao que cada município isoladamente não conseguiria, conforme a fala da entrevista do grupo de interesse Gestores:

Naquela época se trabalhava muito com a ideia de microrregiões de saúde. (G1)

Uma característica apontada em ambos os PIs 2003 e 2016, sobre os municípios da microrregião da Quarta Colônia dizem que: "a maioria são de pequeno porte, com predominância de minifúndio, as quais organizam suas atividades com base no binômio soja e fumo" (Nova Palma, 2003 p. 01; Bertoldo, 2016 p. 01). Desta forma com características similares, se unem, para o direcionamento da assistência em saúde às pessoas com uso de álcool e outras drogas, mas também se fez necessário burocraticamente para atingir o número de habitantes mínimos necessários para abertura do serviço que era de 20 mil habitantes para CAPS AD (Brasil, 2002). Conforme fala do grupo gestores:

A configuração em nível regional na época se dá porque tinha que ter uma determinada quantidade de população e aí só Nova Palma não tinha, e é por isso que foi feito a nível de regional pra chegar a quantidade de população. (G1)

A regionalização ao mesmo tempo em que é estratégia de um cuidado integral que visa suprir demandas em comum de cidades de pequeno porte, apresenta também adversidades para a efetivação do seu objetivo: a distância e a garantia do meio de transporte.

Nota-se que a regionalização tem na distância do município sede, um dificultador da assistência em saúde e na efetivação de um trabalho em rede com os municípios mais longe. A eficácia do matriciamento também é prejudicado e muitas vezes a equipe busca a estratégia de realizá-lo mesmo que por via telefônica a fim que não se perca o vínculo construído com as diversas equipes da rede de cuidado.

A gente procura estar em constante contato com os municípios, isso através do matriciamento, mas muito mais por telefone, porque tem as dificuldades do município de transporte tanto da gente quanto dos municípios do pessoal vir aqui. (G3)

Em compensação os municípios mais próximos são os mais presentes no compartilhamento de casos. Mas, o que chama a atenção são os municípios com extensão territorial que dificulta o acesso do usuário à zona urbana:

A gente tem mais dificuldade com os mais longe mesmo, tem municípios que a extensão territorial é grande, e tem usuários que tem que percorrer vinte quilometros para chegar na cidade para pegar o transporte, então a gente acaba perdendo esses casos, pela dificuldade de deslocamento. (G3) 
O que denota a falta uma logística, uma organização, uma contrapartida/compromisso dos municípios com o tratamento do seu usuário. Observa-se a importância da atenção primária em saúde cumprir com seu papel no território, garantindo o tratamento medicamentoso e terapêutico, tanto quanto como moderador e organizador do cuidado, referenciando ao CAPS AD somente em necessidade de nova consulta, além de ter na equipe do CAPS AD um serviço apoio e assessoria.

O cuidado para tratamento do uso de álcool e outras drogas na região da quarta colônia tem na forma regional uma estratégia de garantia de direitos e acesso ao serviço especializado. Mas como programas e prestadores de serviços necessitam de avaliações periódicas e nesse caso novas contratualizações, saindo do aparato burocrático, alcançando às necessidades reais dos seus usuários.

A partir da configuração regional, duas ações realizadas pelo CAPS AD regional Pe. Otávio Ferrari merecem destaque por que fundamentam a sua implantação na região da quarta colônia: a intersetorialidade e o matriciamento. Tais ações estão presentes nos objetivos dos projetos institucionais do serviço.

"Promover o atendimento integral aos usuários e seus familiares; Promover atividades de capacitação profissional em alcoolismo e drogadição; -Criar mecanismos de vigilância para que sejam identificados os fatores de risco que propiciem o uso abusivo de álcool e outras drogas; Incentivar a inclusão dos usuários no seu contexto social, como trabalho, família e comunidade; Reduzir o índice de alcoolismo e drogadição na região; Fazer integração com a rede de atendimento dos municípios" (Bertoldo, 2016, p. 03).

Observa-se entre os objetivos a proposta de matriciamento, quando cita a promoção de atividade de capacitação profissional e de intersetorialidade quando propõe integração com a rede de atendimento dos municípios. Entre os objetivos citados, dar-se-á ênfase para discussão esses dois itens, que além de citados nas falas dos grupos de interesse do estudo, os demais são comuns às atividades e ações dos CAPS de outras modalidades.

\section{Intersetorialidade e Matriciamento}

Entre os dispositivos da RAPS, o CAPS é considerado como o principal articulador entre os demais serviços, além de estratégico na organização da rede de atenção em saúde mental. Devendo permanecer articulado com a rede de atenção à saúde e com as demais redes sociais que os circundam, de modo a responderem à demanda de assistência no espaço comunitário e de reinserção social (Fidelis, 2018). Essa articulação promove a intersetorialidade, presente como processo de trabalho do CAPS AD Pe. Otávio Ferrari desde a implantação conforme ressaltado no grupo focal dos trabalhadores:

\section{A equipe do CAPS era responsável pela alta. (T3)}

Assim, nota-se que o serviço era o responsável pelo contato com a rede de cuidado do município de origem do usuário. Tal relato vai ao encontro da fala das entrevistas com os gestores:

Essa foi a proposta de se criar um serviço que pudesse atender as pessoas e fazer a vinculação com os municípios, tentar as pessoas que se internassem e então tinha uma equipe que fazia todo esse contato com a referência que contra referência com os municípios. (G1)

Neste sentido, a intersetorialidade é um dos objetivos propostos para implantação do serviço de forma a articular a rede de cuidado do usuário. A noção de intersetorialidade parte de um entendimento de saúde que considera as pessoas em sua totalidade, e com o fim de demonstrar ações resolutivas faz-se necessário parcerias entre diversos setores, estratégia complexa que busca superar a fragmentação das diversas políticas e das diferentes áreas em que são executadas (Brasil, 2009; Leal \& 
Antoni, 2013).

Ainda, contempla a construção de interfaces e diálogos entre setores, as quais suas intenções e ações visem o enfrentamento de problemas sociais complexos, além de superarem a capacidade das políticas públicas enfrentarem de forma solitária, como uma alternativa ou como impulsionador para que se garanta a promoção e prevenção em saúde (Hoff, 2016).

O documento PI 2016 cita como serviços da rede de cuidado em saúde mental: a Atenção Primária da Saúde (APS) formadas pelas Unidades Básicas de Saúde (UBS) e as Estratégias Saúde da Família (ESF); o Serviço de Atenção Integral à Saúde Mental (SERAISM-AD); os Centros de Referência de Assistência Social (CRAS); os Conselhos tutelares; Escolas municipais e estaduais; Delegacia da Polícia Civil; Brigada Militar; Empresa de Assistência Técnica e Extensão Rural (EMATER) e os serviços de Alcoólicos Anônimos (AA). Serviços estes da rede de atenção em saúde e rede socioassistencial que caracterizam um cuidado intersetorial.

Apesar de citados no PI 2016, serviços de saúde e socioassistenciais, nas falas o trabalho intersetorial mais efetivo ocorre entre a Política de Assistência Social, Saúde e Educação. Neste sentido a articulação entre os serviços de saúde e os demais da rede de cuidado da área de abrangência da quarta colônia, visa buscar além de ofertar um cuidado resolutivo e humanizado que trate o sujeito na sua totalidade, promover a prevenção e promoção da saúde na rede regional.

O AA foi a primeira oportunidade que eu tive na minha vida. E na recaída eu busquei o CAPS [...] recaí depois de 20 anos, perdi tudo, e foi aí que eu procurei o serviço aqui e estou me recuperando. (U5)

A gente ficou um tempo sem assistente social, então o CRAS deu um suporte, dentro do que eles conseguiam até por conhecer as famílias, a gente consegue fazer esse trabalho com eles. (G3)

Eu estava pensando no nosso município (município sede) é com a atenção básica mas nos outros municípios é com outros o contato, é Centro de Referência Especializado em Assistência Social (CREAS), a Assistência Social, Núcleo de Apoio à Saúde da Família (NASF), que a gente tem maior contato não é com a Atenção Básica. (T1)

Observa-se que a atenção primária da saúde é o serviço que mais busca a clínica ampliada, mas não se efetiva o cuidado, a equipe consegue um trabalho eficaz com outros serviços, caracterizando assim o apoio matricial com serviços intersetoriais à política de saúde. O apoio matricial, segundo o PI 2016 é realizado por meio de reuniões que acontecem em todos os municípios de cobertura do CAPS AD com intuito na construção de momentos onde se estabelece troca de saberes entre os profissionais, de diferentes serviços de atenção (Bertoldo, 2016).

O Matriciamento ou apoio matricial garante o acesso ao atendimento às pessoas que se encontram à margem do sistema de saúde, desassistidas, sem possibilidades de um atendimento especializado, dessa forma a utilização da APS promove um atendimento qualificado, não sendo necessário o encaminhamento ao serviço especializado de casos leves e moderados (Hirdes \& Scarparo, 2015). O Matriciamento consiste na existência de uma equipe organizacional que oferece o suporte técnico-pedagógico de apoio para uma equipe de referência, ou seja, uma equipe especializada que dê suporte ao serviço no território que é responsável por assistir a população (Iglesias \& Avellar, 2019).

As vantagens de um cuidado de base territorial incluem: conhecimento dos profissionais da situação de vida daquela pessoa (e não somente da doença), a questão do vínculo, a possibilidade de acionar recursos da própria comunidade, a conexão com outros setores - educação, assistência social, programas governamentais, entre outros (Hirdes \& Scarparo, 2015). Na fala das entrevistas dos gestores, o matriciamento foi uma das principais propostas de ações a serem realizadas pelo CAPS AD Pe. Otávio Ferrari, para a sua implantação:

A proposta da criação junto ao Ministério da Saúde foi os CAPS regionais serem apoio matricial para os outros 
municípios [...] para que eles pudessem ajudar os municípios organizarem a sua rede de cuidados. Esta foi a proposta porque a gente precisava recursos para isso, então, a proposta que a gente fez foi continuar as internações pra todos os municípios da regional [...] a gente não conseguiria dar todo o atendimento aos 26 municípios, mas ficava de retaguarda para as equipes dos municípios, além da região da quarta colônia. (G1)

Observa-se a ideia de matriciamento como equipe de retaguarda para a APS:

A gente percebe que de uns dois anos pra cá a gente tem sido mais solicitado quanto a isso aqui na região, foram feitos mais matriciamento e quando a gente vai nos municípios tenta estratégias de como eles vão receber esse usuário de volta. (G3)

O matriciamento na APS da quarta colônia é um instrumento a ser reforçado, sendo que alguns municípios começam a se ver como protagonista do cuidado em território, e outros a ser implementado. O grupo focal dos trabalhadores afirma:

A gente tem conversado seguidamente, tem um bom diálogo de entendimento, a gente recorre aos agentes comunitários de saúde ou com a enfermeira, coordenadora da estratégia... eu percebo que existe uma evolução positiva. (T3)

Percebe-se que o usuário reforça com a ideia de cogestão do tratamento:

Eu vou trato os dentes, pego remédio, quando eu não vou, meu pai vai na ESF... e as receitas pra esses remédios eu pego lá. (U1)

Já alguns municípios mostram resistência, referenciando o tratamento somente ao CAPS AD:

A atenção básica parece que não está estruturada pra isso, a gente sabe tem essa dificuldade de ficar com o paciente também, a gente sabe que é nosso, mas é deles também, mas depois que é nosso, corta o vínculo lá com a atenção básica. (T1)

Nota-se que a atenção primária não realiza a cogestão do cuidado e identifica que álcool e drogas devem ser encaminhados para um serviço especializado na rede, nesse caso, o CAPS AD. Já, o trabalhador percebe a necessidade de um cuidado compartilhado para o cuidado clínico, mas também a APS como organizadora do cuidado, acessando o CAPS AD para novas consultas, apoio nas crises e/ou recaída, além do monitoramento longitudinal:

Tinha que referenciar novamente, porque o álcool e drogas tem uma consequência do uso e que seria uma referência pra atenção básica cuidar esse usuário. (T4)

O matriciamento em saúde mental na APS promove a integração dos profissionais apoiadores especialistas com os profissionais das equipes de referência a partir de práticas interdisciplinares que implicam na qualidade da assistência e na clínica ampliada (Almeida et al., 2020). A assistência em saúde de modo intersetorial torna-se relevante com a efetivação do apoio matricial, já que este último, tenta reverter a lógica do encaminhamento, que pressupõe a hierarquização entre os saberes e a transferência de responsabilidades, para propor a lógica da corresponsabilização. Neste sentido, substitui a relação poder/saber pelo compartilhar de responsabilidades, exigindo que os profissionais decidam juntos sobre o andamento de cada 
caso, bem como sobre seu acompanhamento (Dantas \& Passos, 2018).

O matriciamento aparece, então, como sendo algo a ser reforçado, articulando um cuidado intersetorial entre serviços de diferentes políticas e complexidades. Os usuários desta RAPS, foco deste estudo, têm um endereço em uma região que a cobertura da saúde da família é boa, mas mostra fragilidades. Onde a atenção básica faz parte do tratamento em território, por vezes, sendo a porta de entrada, por vezes monitorando, coordenando, organizando o cuidado e demonstrando a necessidade de fortalecer o cuidado compartilhado.

\section{Considerações Finais}

Com a premissa de emergir as potencialidades e os obstáculos identificados pelos diferentes grupos de interesse inseridos no CAPS regional para o fortalecimento da RAPS a que está inserido, se destacou que a configuração regional, além de ser uma estratégia de gestão para prestação de serviço que contemple as três complexidades de gestão do cuidado, busca suprir uma demanda comum de municípios de pequeno porte. Se observou que há ganhos, mas há a necessidade de novas repactuações a cada nova gestão, para que o dispositivo continue sustentável e correspondendo as demandas da RAPS a que está inserido. Também é preciso implementar as tecnologias já desenvolvidas e fortalecer a atenção primária da região como a organizadora do cuidado compartilhado em território.

Diante do atual contexto macropolítico do país, de retrocesso de 30 anos de conquistas da política de saúde mental, é necessário incentivar estudos que demonstrem experiências valiosas como esta, de um CAPS AD na configuração regional, que implica em transformações sociais e culturais que potencializa um cuidado humanizado em saúde mental na RAPS em âmbito regional. Bem como se torna de grande valia quando confronta o atual cenário onde o governo federal aprova duas medidas que estagnam o progresso gradual referente ao tratamento em saúde mental, que vinha alcançando a reforma psiquiátrica.

Cita-se a nota técnica do último dia 04 de fevereiro de $\mathrm{N}^{\circ}$ 11/2019 onde inclui na RAPS as comunidades terapêuticas e aprova o retorno, com aumento de leitos, dos hospitais psiquiátricos, ambos com incentivo federal, que realizam tratamentos de forma segregada, isolada e sem supervisão técnica na sua maioria. Somando a isto, é aprovada recentemente a internação involuntária por meio da Lei $\mathrm{N}^{\circ} 13.840 / 2019$, medida que desrespeita a singularidade da pessoa, quando autoriza que desobriga o pedido de internação por familiares ou responsáveis, permitindo que estas também possam ser realizadas profissionais da linha de cuidado da saúde e sócia assistenciais, além de autoridades.

Tais medidas dão margem a ações de coerção, repressão, criminalização e marginalização do usuário de drogas. Por fim, há que se fortalecer, há que resistir, desmistificar, sendo um dos meios para tal, a publicação e divulgação de resultados dos estudos em saúde mental que fortalecem a política de saúde mental com ênfase na humanização, serve de referência para novas pesquisas e produção de conhecimentos.

\section{Referências}

Almeida, D. R, Soares, J. N. C, Dias, M. G, Rocha F. C, Andrade Neto G. R \& Andrade, D. L. B. (2020) Care for carriers of mental disorder in primary care: an interdisciplinary and multiprofessional practice. R. pesq.: cuid. fundam. online. 12(1), 420-425.

Amarante, P. \& Nunes, M. O. (2018) A reforma psiquiátrica no SUS e a luta por uma sociedade sem manicômios. Ciência \& Saúde Coletiva. 23(6), 20672074.

Amarante, P. (2010). Psiquiatria Social e Reforma Psiquiátrica. (4a ed.), Fiocruz.

Backes, D. S., et al. (2011) Grupo focal como técnica de coleta e análise de dados em pesquisas qualitativas. O mundo da saúde. 35(4), 438-442.

Bertoldo, N. R. (2016) Projeto Institucional do Centro de Atenção Psicossocial Regional Padre Otávio Ferrari. RS, Nova Palma, 2016. 
Brasil. Decreto $n^{\circ} 7.508$, de 28 de junho de 2011. Regulamenta a Lei no 8.080, de 19 de setembro de 1990, para dispor sobre a organização do Sistema Único de Saúde - SUS, o planejamento da saúde, a assistência à saúde e a articulação interfederativa, e dá outras providências. Diário Oficial da União de 29.06.2011. Brasília, DF, 2011b. http://www.planalto.gov.br/ccivil_03/_ato2011-2014/2011/decreto/d7508.htm.

Brasil. Lei no 10.216, de 6 de abril de 2001. Dispõe sobre a proteção e os direitos das pessoas portadoras de transtornos mentais e redireciona o modelo assistencial em saúde mental. Diário Oficial da República Federativa do Brasil. Brasília, DF, 2001a. [citado em 2017 set. 15]. Disponível em: http://hpm.org.br/wp-content/uploads/2014/09/lei-no-10.216-de-6-de-abril-de-2001.pdf.

Brasil. Ministério da Saúde. Nota Técnica No 11 de o4 de fevereiro de 2019. Esclarecimentos sobre as mudanças na Política Nacional de Saúde Mental e nas Diretrizes da Política Nacional sobre Drogas. Coordenação-Geral de Saúde Mental, Álcool e Outras Drogas Brasília, DF, 2019. http://pbpd.org.br/wpcontent/uploads/2019/02/0656ad6e.pdf.

Brasil. Ministério da Saúde. Conselho Nacional de Saúde. Diretrizes e normas regulamentadoras sobre pesquisa envolvendo seres humanos. Resolução 466. Brasília: CNS, 2012.

Brasil. Ministério da Saúde. Lei n. ${ }^{\circ}$ 10.708, de 31 de julho de 2003, institui o auxílio reabilitação para pacientes egressos de internações psiquiátricas 103 (Programa De Volta Para Casa). Diário Oficial da União. Brasília, DF, 2003.

Brasil. Ministério da Saúde. O SUS de A a Z: garantindo saúde nos municípios. Brasília: Ministério da Saúde: 2009. http: http://bvsms.saude.gov.br/bvs/publicacoes/sus_az_garantindo_saude_municipios_3ed_p1.pdf.

Brasil. Ministério da Saúde. Portaria MS/GM no 336 de 19 de janeiro de 2002. Estabelece que os Centros de Atenção Psicossocial poderão constituir-se nas seguintes modalidades de serviços: CAPS I, CAPS II e CAPS III, definidos por ordem crescente de porte/complexidade e abrangência populacional. Brasília: Ministério da Saúde, 2002. http://bvsms.saude.gov.br/bvs/saudelegis/gm/2002/prt0336_19_02_2002.html. Acessado em 19 jan. 2018.

Brasil. Ministério da Saúde. Portaria No- 3.090, de 23 de dezembro de 2011. Estabelece que os Serviços Residenciais Terapêuticos (SRTs), sejam definidos em tipo I e II, destina recurso financeiro para incentivo e custeio dos SRTs, e dá outras providências. Brasília, DF, 2011a.

Brasil. Ministério da Saúde. Portaria No 95, de 26 de janeiro de 2001. Da Norma Operacional da Assistência à Saúde - NOAS-SUS 01/2001 que amplia as responsabilidades dos municípios na Atenção Básica, define o processo de regionalização da assistência, cria mecanismos para o fortalecimento da capacidade de gestão do Sistema Único de Saúde e procede à atualização dos critérios de habilitação de estados e municípios. Ministério da Saúde. Brasília, DF, 2001b.: http://bvsms.saude.gov.br/bvs/saudelegis/gm/2001/prt0095_26_01_2001.html.

Brasil. Ministério da Saúde. SAS/DAPES. Coordenação Geral de Saúde Mental, Álcool e Outras Drogas. Saúde Mental em Dados - 12, Ano 10, $\mathrm{n}^{\circ}$ 12, outubro de 2015. Brasília, 2015. Informativo eletrônico de dados sobre a Política Nacional de Saúde Mental. 48p. www.saude.gov.br/bvs/saudemental.

Brasil. Portaria GM/MS n 3.088, de 23 de dezembro de 2011. Institui a Rede de Atenção Psicossocial para pessoas com sofrimento ou transtorno mental e com necessidades decorrentes do uso de crack, álcool e outras drogas, no âmbito do Sistema Único de Saúde (SUS). Brasília, DF, 2011c. http://bvsms.saude.gov.br/bvs/saudelegis/gm/2011/prt3088.

Dantas, N. F. \& Passos, I. C.F. (2018) Apoio matricial em saúde mental no SUS de Belo Horizonte: perspectiva dos trabalhadores. Trabalho, Educação, Saúde. 16(1), 201-220.

Fidelis, A. C. (2018) Sentido do cuidado em saúde mental: sobre a rede de atenção psicossocial do Sistema Único de Saúde. Trabalho, Educação, Saúde. 16(2), 561-582.

Figueiredo, M. D. \& Campos, R. O. (2009) Saúde Mental na atenção básica à saúde de Campinas, SP: uma rede ou um emaranhado? Ciência \& Saúde Coletiva. 14(1), 129-138.

Guba, E. G. \& Lincoln, Y. S. (2011) Avaliação de quarta geração / tradução Beth Honorato. Editora da Unicamp.

Hirdes, A. \& Scarparo, H. B. K. (2015) O labirinto e o minotauro: saúde mental na Atenção Primária à Saúde. Ciência saúde coletiva. 20(2), 383-393.

Hoff, L. R. (2016) Público e Privado na política de saúde mental: inflexões de “um mundo à parte”. EDIPUCRS.

IBGE. Instituto Brasileiro de Geografia e Estatística. CENSO 2010. Nova Palma. https://cidades.ibge.gov.br/brasil/rs/nova-palma/panorama.

Iglesias, A. \& Avellar, L. Z. (2019) Matriciamento em Saúde Mental: práticas e concepções trazidas por equipes de referência, matriciadores e gestores. Ciênc. saúde coletiva. 24(4), 1247-1254.

Kantorski, L. P. et.al. (2011) Avaliação de Quarta Geração: aplicação no campo da saúde mental. Desafios da avaliação de programas e serviços em saúde: novas tendências e questões emergentes. Campos, R.O. \& Furtado, J. P. (Org.). Editora da Unicamp, 2011.

Kantorski, L. P. et.al. (2009) Avaliação de Quarta Geração: contribuições metodológicas para avaliação de serviços de saúde mental. Interface Comunicação, Saúde, Educação. 13(31), $343-55$.

Leal, B. M. \& Antoni, C. (2013) Os Centros de Atenção Psicossocial (CAPS): estruturação, interdisciplinaridade e intersetorialidade. Aletheia, Canoas, (40), $87-101$.

Nova Palma. Prefeitura Municipal De Nova Palma. (2003) Projeto Institucional do Centro de Atenção Psicossocial Regional Padre Otávio Ferrari. RS, Nova Palma.

Nova Palma. Prefeitura Municipal De Nova Palma. (2019) Dados Gerais do Município de Nova Palma. RS, Nova Palma.

Righi, L. \& Gonçalves, C. (2018) Cartografia e Hermenêutica nos Enlaces da Pesquisa Participativa. Revista Polis e Psique. 8(1), 132-143. 
Research, Society and Development, v. 11, n. 2, e2311225304, 2022

(CC BY 4.0) | ISSN 2525-3409 | DOI: http://dx.doi.org/10.33448/rsd-v11i2.25304

Rio Grande Do Sul. Secretaria da Saúde. Plano Estadual de Saúde: 2016/2019. Grupo de Trabalho de Planejamento, Monitoramento e Avaliação da Gestão (Org.) Porto Alegre, 2016a. 228 p. Il. Disponível em: http://www.saude.rs.gov.br/upload/arquivos/201701/05153251-pes-2016-2019-sesrs.pdf. Acessado em 28 jan. 2019.

Rio Grande Do Sul. Secretaria Estadual de Saúde (SES). Resolução nº 555/2012. Comissão Intergestores Bipartite 2016. SES, 2016 b.

Saad, D., S., Saad, D. S., \&Machado, M. V. M. (2012) Quarta Colônia de imigração italiana, patrimônio cultural e turismo no planalto central do Rio Grande do Sul, no Sul do Brasil. Revista América Patrimônio. 4(1), 55-68.

Souza, F. S. P. \& Jorge, M. S. B. (2018) O retorno da centralidade do hospital psiquiátrico: retrocessos recentes na política de saúde mental. Trabalho, Educação, Saúde. 17(1), e0017201.

Teixeira, C. F. (2002) Promoção e vigilância da saúde no contexto da regionalização da assistência à saúde no SUS. Cadernos Saúde Pública. 18(supl), 153162. 